\title{
Robot-assisted thoracoscopic surgery versus thoracotomy for c-N2 stage NSCLC: short-term outcomes of a randomized trial
}

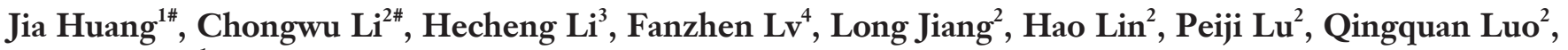 \\ Wenrong $\mathrm{Xu}^{1}$ \\ ${ }^{1}$ Jiangsu Key Laboratory of Medical Science and Laboratory Medicine, School of Medicine, Jiangsu University, Zhenjiang 212013, China; ${ }^{2}$ Shanghai \\ Lung Cancer Center, Shanghai Chest Hospital, Shanghai Jiao Tong University, Shanghai 200030, China; ${ }^{3}$ Department of Thoracic Surgery, Ruijin \\ Hospital, Shanghai Jiao Tong University School of Medicine, Shanghai 200030, China; ${ }^{4}$ Department of Thoracic Surgery, The Affiliated Huadong \\ Hospital of Fudan University, Shanghai 200030, China \\ Contributions: (I) Conception and design: J Huang, Q Luo, W Xu; (II) Administrative support: C Li, Q Luo; (III) Provision of study materials or \\ patients: H Lin, P Lu; (IV) Collection and assembly of data: J Huang, L Jiang; (V) Data analysis and interpretation: C Li, H Lin; (VI) Manuscript \\ writing: All authors; (VII) Final approval of manuscript: All authors. \\ "These authors contributed equally to this work. \\ Correspondence to: Wenrong Xu. Jiangsu Key Laboratory of Medical Science and Laboratory Medicine, School of Medicine, Jiangsu University, \\ Zhenjiang 212013, China. Email: xuwenrong@126.com.
}

Background: Safety and short-term efficacy of robot-assisted thoracoscopic surgery (RATS) for early-stage non-small cell lung cancer (NSCLC) have been previously proven; however, RATS for N2 stage NSCLC was barely evaluated. The aim of this randomized controlled trial (RCT) was to explore the short-term outcome of RATS for cN2 stage NSCLC.

Methods: Total of 113 patients who were diagnosed with clinically single cN2 stage NSCLC were enrolled and randomly assigned to RATS and thoracotomy groups. The patients in RATS group were treated by lobectomy and mediastinal lymph node dissection using the da Vinci Surgical System, while the patients in thoracotomy group underwent lobectomy and mediastinal lymph node dissection from. And, short-term outcomes were analyzed statistically.

Results: The data from 113 subjects (58 in RATS and 55 in thoracotomy groups) were eligible for analyses. Five patients who received robot-assisted lobectomy initially was converted intraoperatively to open operation due to extensive pleural adhesion and equipment issues. And, one subject underwent robotassisted surgery was died preoperatively due to pulmonary embolism. Compared with thoracotomy, RATS was associated with less intraoperative blood loss $(86.3 \pm 41.1$ vs. $165.7 \pm 46.4 \mathrm{~mL}, \mathrm{P}<0.001)$, median chest duration ( 4 vs. $5, \mathrm{P}<0.01$ ), visual analog scores at postoperative day one to five $(\mathrm{P}<0.001)$, and slightly fewer incidence of postoperative complications. Also, both surgical approaches revealed comparable drainages and nodal harvest. The cancer residual margins occurred in one subject in RATS group and three patients in thoracotomy group $(\mathrm{P}=0.56)$. However, overall cost of subjects underwent RATS was higher than those received thoracotomy $(100,367 \pm 19,251$ vs. $82,002 \pm 20,434, \mathrm{P}<0.001)$.

Conclusions: Present study proves that the feasibility and safety of RATS lobectomy to treat patients with cN2 stage NSCLC, and it should be superior to thoracotomy due to lesser intraoperative blood loss.

Keywords: Da Vinci robot surgery system; thoracotomy; N2; non-small cell lung cancer (NSCLC)

Submitted Sep 05, 2019. Accepted for publication Oct 21, 2019.

doi: $10.21037 /$ tlcr.2019.11.31

View this article at: http://dx.doi.org/10.21037/tlcr.2019.11.31 


\section{Introduction}

Robot-assisted thoracoscopic surgery (RATS) was first used for non-small cell lung cancer (NSCLC) in 2002 $(1,2)$ and had been rapidly gaining popularity worldwide attributing to its minimal invasiveness. The increasing data show that RATS lobectomy is safe and effective for treating patients with early-stage NSCLC, but fewer and retrospective studies on RATS to treat patients with c-N2 NSCLC require robust evidence to evaluate its safety and effectiveness (3). Thus, this multicenter randomized controlled trial (RCT) was designed in China to identify the safety and effectiveness of RATS for treating patients with c-N2 NSCLC by compared with thoracotomy, which is golden standard for surgical therapy of c-N2 NSCLC patients. The required follow-up period has not yet been reached; data from secondary endpoints were collected and analyzed here to estimate short-term perioperative outcomes of RATS.

\section{Methods}

\section{Study design}

This noninferiority, phase 3, multi-center RCT was approved by the ethics board of Shanghai Chest Hospital (KS1735) and registered at Chinese Clinical Trial Registry (ChiCTR-INR-17012777). This study was launched in January 2016.

\section{Subjects}

The patients aged from 18 to 75 years who were clinically diagnosed c-N2 NSCLC according to American Joint Committee on Cancer Tumor-Node-Metastasis classification exhibited as a suspicious pulmonary lesion with enlarged mediastinal lymph nodes (diameter bigger than $1 \mathrm{~cm}$ on computed tomography scan) were considered as candidate (4). The subjects enrolled in this study must enable to give written informed consent, and their organ function must be adequate to tolerate pulmonary resection. The patients were ineligible if they accompanied with invasion into neighboring organs (such as hilum), extensive pleural adhesion, earlier thoracotomy, highdose radiation on the chest, history of other malignancies in the past 5 years except for nonmelanoma skin cancer, cervix cancer in situ, or early-stage prostate cancer. The ineligibility criteria also included predicted postoperative forced expiratory volume in 1 second (FEV1) or diffusing capacity of lung for carbon monoxide value less than $40 \%$, pregnant or lactating female patients, inability to obtain consent. And, intraoperative pleural adhesion or technical challenge to achieve hemostasis needed conversion from RATS to thoracotomy. Furthermore, the following criteria were defined as exclusion in present study, including occult pleural metastasis; major protocol violation; clinician decides the patient should not continue the trail according to individual condition; patients withdraw from the trial; histologic finding is not NSCLC.

All participants gave written informed consent according to International Conference on Harmonisation (5), and then, were registered by the local investigator in each participating institution on the trial's website to ensure allocation concealment. Following a list of randomization number generated in the trial statistician's computer with stratification for the participating center, the subjects enrolled in present study were randomly and equally assigned to RATS and thoracotomy groups. The allocation was done by telephone by the trial coordinator. Neither subjects nor any investigators were masked to treatment allocation.

\section{Preoperative work-ups and perioperative care, surgical procedure}

Before the operation, history, physical examination, hematologic and biochemical tests, pulmonary function test, computed tomography of chest and abdomen, brain magnetic resonance imaging and diagnostic bronchoscopy were carried out according to the protocol. In all cases, a radical lobectomy combined with mediastinal lymph node dissection was performed. The RATS was operated using the da Vinci Surgical System (Intuitive Surgical, Sunnyvale, CA, USA) and followed the strict definition of the Cancer and Leukemia Group B 39802, which included anatomic lobectomy, visualization only by thoracoscope and non-ribspreading technique (6) (Figures 1,2). The number of ports as defined in the trial.

On the other hand, subjects in the control group underwent conventional lobectomy with a rib-spreading thoracotomy of 15 to $20 \mathrm{~cm}$. In present study, the hilar and mediastinal lymph nodes were routinely dissected, and a minimum of three mediastinal lymph nodes (N2) stations was harvested. In detail, station 2, 3a, 4, 7, 8, 9, 10, and 11 lymph nodes were dissected in right lobectomy, while station 4, 5, 6, 7, 8, 9, 10 and 11 lymph nodes were dissected in left lobectomy. The occurrence of macroscopic residual 


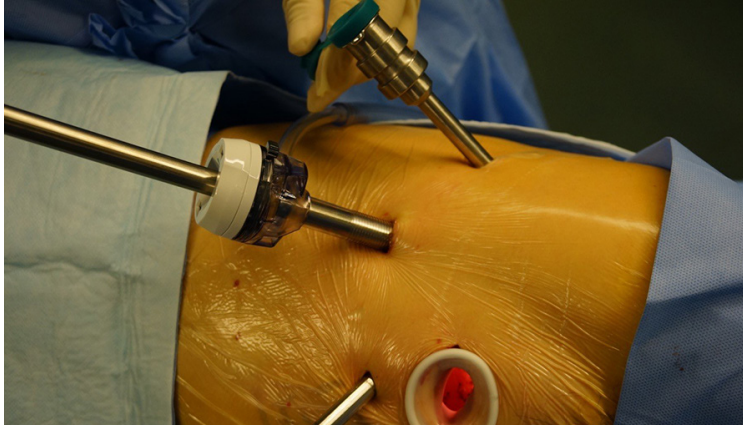

Figure 1 The incision of RATS.

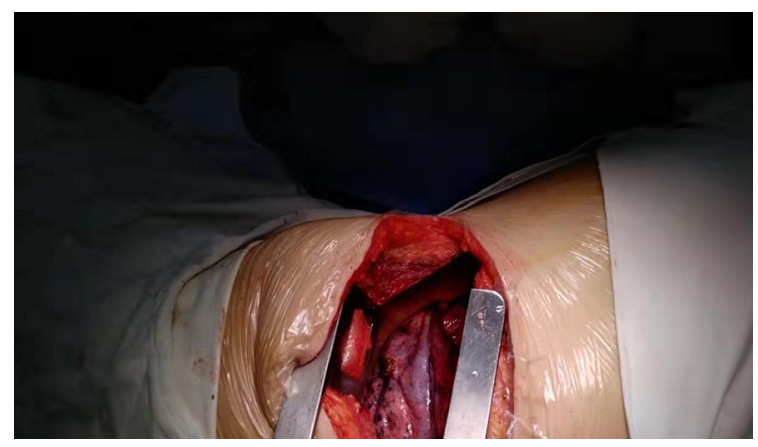

Figure 2 The incision of thoracotomy.

cancer in resection margin or unresectable malignant lymphadenopathy was defined as incomplete resection. After surgery, histologic examination was routinely performed to identify at least the histologic type, tumor size, the extent of invasion (pleural, neural, lymph vascular, and bronchial involvement), adequacy of surgical margins, and lymph node metastatic status. Within the first 2 years after treatment, patients were reviewed in outpatient clinic in 3-month intervals. Then, the surveillance was scheduled on a 6-month basis for the next 3 years.

\section{Statistical analysis}

Before the operation, clinical characteristics of patients were recorded, including gender, smoking history, comorbidities, FEV1, diffusing capacity for carbon monoxide (DLCO) and tumor location. And, these characteristics were submitted to analyze to identify whether baseline factors were well balanced. In addition, data related to operation were collected and compared statistically, including intervention, operative time (minutes), intraoperative blood loss (mL), chest tube duration (day), drainage at postoperative day one $(\mathrm{ml})$ and total drainage $(\mathrm{mL})$, length of hospital day (LOS), death (within 28 days), complications, visual analogue score (VAS) at postoperative day one to five (POD1-5) and overall cost $(¥)$. Furthermore, pathologic variables were also recorded and analyzed, including histologic subtype, tumor size, pathologic stage, surgical margin, sampled lymph node stations, number of lymph nodes and number of $\mathrm{N} 2$ lymph nodes. Data from variables related to perioperative care were not collected because of their potential mild variation among the three centers. To verify difference of complications, grading was used and described as follows. Postoperative hemorrhage of up to $750 \mathrm{~mL}$ was grade 1,750 to $1,500 \mathrm{~mL}$ was grade $2,1,500$ to $2,000 \mathrm{~mL}$ was grade 3 , and more than $2,000 \mathrm{~mL}$ was grade 4 . Pneumonia was graded based on CURB-65 score [confusion, BUN $>19 \mathrm{mg} / \mathrm{dL}$, respiratory rate $>30$, systolic blood pressure (BP) $<90 \mathrm{mmHg}$ or diastolic $\mathrm{BP} 60 \mathrm{mmHg}$, age $>65$ ] with score 0 to 1 being mild, score of 2 being moderate, and score of 3 or more being severe. Air leak was considered mild when present only on cough, moderate when present with speaking and severe when present with breathing. All the other complications were confirmed by laboratory or radiologic findings.

Statistical analysis was performed by SPSS 16.0 (SPSS Inc. Chicago, IL, USA). The differences between two groups were analyzed using Student $t, \mathrm{c} 2$, and Fisher exact tests or Wilcoxon rank-sum test. Data were shown as median $\left(25^{\text {th }}\right.$ to $75^{\text {th }}$ percentile) when they were not normally distributed. Patients who suffered from severe adverse events may be taken off the trial for safety but will continue their follow-up for analysis of primary endpoints. The two-sided $\mathrm{P}$ value of less than 0.05 was considered as statistically significant.

\section{Results}

This trial was performed in three leading thoracic referral centers in China from January 2016 to December 2018. Among total of 113 recruited patients, 90 participants were from Shanghai Chest Hospital, 13 patients were from Shanghai Ruijin Hospital, and 10 subjects were from Shanghai Huadong Hospital. By a list of randomization number, 58 subjects were assigned to the RATS group, and 55 patients were assigned to axillary thoracotomy group. In present study, RATS surgery was converted to thoracotomy in five subjects due to extensive pleural adhesion (two cases) and equipment issues (three cases). Thus, data from 53 cases in RATS and 55 cases in axillary thoracotomy were collected 
Table 1 Clinical characteristics of patients

\begin{tabular}{|c|c|c|c|}
\hline Characteristic & $\begin{array}{l}\text { RATS } \\
(n=58)\end{array}$ & $\begin{array}{l}\text { Axillary thoracotomy } \\
\qquad(\mathrm{n}=55)\end{array}$ & $P$ value \\
\hline Sex, n (\%) & & & 0.98 \\
\hline Male & $41(70.7)$ & 39 (70.9) & \\
\hline Female & $17(29.3)$ & $16(29.1)$ & \\
\hline Age, $y, \pm S D$ & $61.9 \pm 9.0$ & $60.6 \pm 7.4$ & 0.40 \\
\hline Smoking history, n (\%) & $28(48.3)$ & $30(54.5)$ & 0.51 \\
\hline \multicolumn{4}{|l|}{ Comorbidities, n (\%) } \\
\hline Hypertension & $16(27.6)$ & $14(25.5)$ & 0.80 \\
\hline Diabetes mellitus & $3(5.2)$ & $5(9.1)$ & 0.66 \\
\hline History of MI or CVA & $4(6.9)$ & $4(7.3)$ & 1.00 \\
\hline Other comorbidities & $2(3.4)$ & $3(5.5)$ & 0.95 \\
\hline No comorbidities & $38(65.5)$ & $36(65.5)$ & 1.00 \\
\hline FEV1\% & 90.3 & 89.5 & 0.80 \\
\hline DLCO\% & 96.5 & 91.1 & 0.13 \\
\hline Tumor location & & & 0.57 \\
\hline Right upper & $24(41.4)$ & $23(41.8)$ & \\
\hline Right middle & $2(3.4)$ & $1(1.8)$ & \\
\hline Right lower & $19(32.8)$ & $15(27.3)$ & \\
\hline Left upper & $4(6.9)$ & $9(16.4)$ & \\
\hline Left lower & $9(15.5)$ & $7(12.7)$ & \\
\hline
\end{tabular}

Data are No. (\%) of patients, unless otherwise noted. RATS, robotic-assisted thoracic surgery; SD, standard deviation; FEV1, forced expiratory volume in 1 second; MI, myocardial infarction; CVA, cerebrovascular accident.

to analysis. The mean age of patients was 61.9 years in the RATS group and 60.6 years in the axillary thoracotomy group $(\mathrm{P}=0.40)$. The significant difference was undetectable in term of gender, body surface, smoking status, pulmonary disorders, and other comorbidities (Table 1). In other word, baseline factors were well balanced between the two groups.

The distribution of different pulmonary resections in RATS group was comparable to those in axillary thoracotomy group $(\mathrm{P}=0.57)$. In all cases, the mediastinal lymph node was completely dissected. The median operative time was 108 minutes in the RATS group and 103 minutes in axillary thoracotomy group $(\mathrm{P}=0.41)$. However, aid of da Vinci Surgical System led to lower intraoperative blood loss $(\mathrm{P}<0.001)$, chest tube duration $(\mathrm{P}<0.01)$ and VAS at postoperative day one to five (POD1-5) $(\mathrm{P}<0.001)$.
Addition, robotic aid generated lower pleural drainage at postoperative day one and total pleural drainage $(\mathrm{P}=0.05)$. The median days of hospitalization in RATS patients were slight less than those in axillary thoracotomy subjects, but statistical significance was undetected. Apart from one subject who died of pulmonary embolism, no operationrelated death was documented for the whole cohort. As shown in Table 2, morbidities of postoperative complications in subjects who underwent RATS were not statistically less than those in participants underwent axillary thoracotomy $(\mathrm{P}=0.23)$.

Further analyses that included the five conversions in the RATS group were performed, and the results were in line with those that excluded the conversions (Table 2). Postoperative examination showed that adenocarcinoma was dominant histologic subtype in both groups, followed by squamous cell carcinoma. Statistical difference was not present in the distribution of pathologic stages $(\mathrm{P}=0.72)$. Incidental stage III disease was identified in 20 patients $(35.1 \%)$ in RATS group and $26(49.0 \%)$ subjects in axillary thoracotomy group, and one stage IV patient in RATS group was intraoperatively found to be adenocarcinoma of with pleural seeding. Moreover, cancer residual margins were found in one subject who underwent RATS and three patients who underwent axillary thoracotomy $(\mathrm{P}=0.56)$. Both surgical approaches generated comparable outcomes in sampled lymph node station $(\mathrm{P}=0.31)$, number of lymph node $(\mathrm{P}=0.79)$ and number of $\mathrm{N} 2$ lymph nodes $(\mathrm{P}=0.38)$ (Table 3).

\section{Discussion}

Earlier evaluation of robotic-assisted lobectomy for lung cancer has documented that surgical robot can confer decreased incidence of complications and enhanced outcomes of various statistical indictors during intraoperative and perioperative period (6-16). Park et al. found that robotic surgery holds unique advantages for patients suffered from locally advanced NSCLC and achieve disease-free survival rate and overall survival rate similar to open surgery (17). Another study showed that surgical robot could remove $9.4 \mathrm{~cm}$ lung cancer tissue under the condition of negative incision margin and thorough systematic lymph node dissection (18). Pieces of evidence above mentioned suggested that RATS should be feasible for local advanced NSCLC. However, feasibility of RATS for c-N2 NSCLC needs to be evaluated. Thus, outcomes of RATS lobectomy for c-NSCLC were compared to axillary thoracotomy in 
Table 2 Operation-related variables and morbidity within 28 days

\begin{tabular}{|c|c|c|c|}
\hline Variable & RATS $(n=58)$ & Axillary thoracotomy $(n=55)$ & $P$ value \\
\hline Intervention & & & 0.57 \\
\hline Lobectomy & 53 & 50 & \\
\hline Bilobectomy & 4 & 2 & \\
\hline Sleeve lobectomy & 1 & 2 & \\
\hline Pneumonectomy & 0 & 1 & \\
\hline Operative time, min, mean \pm SD & $108 \pm 39$ & $103 \pm 30$ & 0.41 \\
\hline Blood loss, $\mathrm{mL}$, mean $\pm \mathrm{SD}$ & $86.3 \pm 41.1$ & $165.7 \pm 46.4$ & $<0.001$ \\
\hline Chest tube duration ${ }^{\mathrm{a}}, \mathrm{d}$ [range] & $4[2-63]$ & $5[3-66]$ & $<0.01$ \\
\hline POD1 drainage, mL [range] & 300 [95-840] & 320 [50-970] & 0.50 \\
\hline Total drainage, $\mathrm{mL}$ [range] & $820[220-2,460]$ & $960[320-4,630]$ & 0.05 \\
\hline LOS, d [range] & $10[7-31]$ & $11[6-44]$ & 0.07 \\
\hline Mortality, n (\%) & $1(1.7)^{b}$ & $0(0)$ & 1.00 \\
\hline \multicolumn{4}{|l|}{ Complications, n (\%) } \\
\hline Any complications & $16(27.6)$ & $21(38.2)$ & 0.23 \\
\hline pulmonary embolism & $1(1.7)$ & $0(0)$ & 1.00 \\
\hline Hemorrhage required reoperation & $1(1.7)$ & $1(1.8)$ & 1.00 \\
\hline BPF & $3(5.2)$ & $1(1.8)$ & 0.65 \\
\hline Esophagus fistula & $0(0)$ & $1(1.8)$ & 0.49 \\
\hline ARDS & $0(0)$ & $1(1.8)$ & 0.49 \\
\hline Pneumonia & $3(5.2)$ & $6(10.9)$ & 0.44 \\
\hline Prolonged air leak & $4(6.9)$ & $6(10.9)$ & 0.68 \\
\hline Atrial arrhythmia & $2(3.4)$ & $3(5.5)$ & 0.95 \\
\hline Chest tube reinsertion & $2(3.4)$ & $3(5.5)$ & 0.95 \\
\hline Chylothorax & $3(5.2)$ & $0(0)$ & 0.24 \\
\hline Recurrent nerve injury & $1(1.7)$ & $4(7.3)$ & 0.33 \\
\hline Others & $1(1.7)$ & $2(3.6)$ & 0.96 \\
\hline \multicolumn{4}{|l|}{ VAS, mean \pm SD } \\
\hline POD1 & $5.9 \pm 1.4$ & $7.0 \pm 1.2$ & $<0.001$ \\
\hline POD2 & $5.4 \pm 1.3$ & $6.9 \pm 1.1$ & $<0.001$ \\
\hline POD3 & $5.0 \pm 1.4$ & $6.2 \pm 1.2$ & $<0.001$ \\
\hline POD4 & $4.1 \pm 1.4$ & $5.4 \pm 1.2$ & $<0.001$ \\
\hline POD5 & $3.7 \pm 1.2$ & $4.8 \pm 1.4$ & $<0.001$ \\
\hline Overall cost, $¥$, mean \pm SD & $100,367 \pm 19,251$ & $82,002 \pm 20,434$ & $<0.001$ \\
\hline
\end{tabular}

Data are No. (\%) of patients, unless otherwise noted. ${ }^{\text {, }}$, two patients in RATS group and two patients in thoracotomy group were discharged with chest tube; ${ }^{b}$, this patient died of pulmonary embolism. RATS, robotic assisted thoracic surgery; POD 1, postoperative day one; LOS, length of hospital stay; BPF, bronchopleural fistula; ARDS, acute respiratory distress syndrome; VAS, visual analog score. 
Table 3. Pathologic Variables

\begin{tabular}{|c|c|c|c|}
\hline Variable & RATS $(n=58)$ & axillary thoracotomy $(n=55)$ & P Value \\
\hline Adenocarcinoma & $42(72.4)$ & $33(60.0)$ & \\
\hline Squamous carcinoma & $9(15.5)$ & $17(30.9)$ & \\
\hline Large cell carcinoma & $3(5.2)$ & $1(1.8)$ & \\
\hline Others & $3(5.2)$ & $2(3.6)$ & \\
\hline Tumor size, cm & $3.27 \pm 1.38$ & $3.64 \pm 1.43$ & 0.17 \\
\hline Pathologic stage & & & 0.72 \\
\hline IA & $10(17.3)$ & $10(18.2)$ & \\
\hline IIB & $14(24.1)$ & $10(18.2)$ & \\
\hline IIIA & $14(24.1)$ & $19(34.6)$ & \\
\hline IIIB & $6(10.3)$ & $7(12.8)$ & \\
\hline IV & $2^{b}(3.4)$ & $2(3.5)$ & \\
\hline Surgical margin $^{c}$ & & & 0.56 \\
\hline Ro & $56(98.2)$ & $50(94.3)$ & \\
\hline $\mathrm{R} 1$ & $1(1.8)$ & $3(5.7)$ & \\
\hline Sampled LN stations & $7.0 \pm 1.1$ & $6.8 \pm 1.4$ & 0.31 \\
\hline
\end{tabular}

Data are No. (\%) of patients, unless otherwise noted. ${ }^{\text {, }}$, histologic subtype was regrouped as adenocarcinoma, squamous carcinoma, and others; ${ }^{\text {b }}$, the stage IV patient was found to be adenocarcinoma with pleural seeding intraoperatively; ${ }^{c}$, benign disease were excluded. RATS, robotic-assisted thoracic surgery; LN, lymph node.

this multicenter RCT.

This trial revealed that usage of da Vinci Robot Surgical System resulted in lesser intraoperative blood loss, which reflects the difficulty and proficiency of the operation, as well as the severity of the trauma. This advanced outcome should attribute to better immunization and full exposure of anatomical structures and usage of disposable cutting and suturing instruments.

The main complications occurred in two cohorts were a pulmonary infection, atrial fibrillation, and air leakage. Earlier studies showed that RATS was associated with relieving pain, reduced phlegm, diminished inflammatory factors and reduced pulmonary infection. Moreover, clearer $3 \mathrm{D}$ visualization and exposure under RATS enable to reduce the damage of blood vessels and lung tissues, thereby, reducing the chances of atrial fibrillation and air leakage. However, the abovementioned complication occurred in patients who underwent RATS surgery and displayed comparable incidence to those underwent axillary thoracotomy, which could be variance of small samples.

During lobectomy for lung cancer, the lymph node was previously suggested to be sampled according to different lobe. Only suspected metastatic lymph nodes should be excised and fast-frozen pathological assay to determine the extent of dissection. However, complexity of lymphatic drainage in the lung could lead to micro-metastasis and jumping metastasis, therefore, systematic lymph nodes dissection is a preferred choice. And, lymph node sampling 
did not improve the complications of surgery. So, direct dissection of systematic mediastinal lymph node was performed in both surgical operations.

The thoroughness of lymph node dissection is pivotal to the comprehensive treatment of lung cancer. It can stage, judge the prognosis, guide the next step of treatment, improve the local control rate, and prolong the diseasefree survival time. However, capability of surgical robot dissects thoroughly mediastinal lymph nodes comparable to thoracotomy has always been the focus of debate, especially to the cases of cN2 NSCLC. An earlier cadaver study indicated that thorough lymph node dissection was carried out in VATS followed by conventional thoracotomy (19). Another study showed that only $2 \%$ to $3 \%$ of the remaining lymph nodes were cleaned by VATS and then further thoracotomy (20). Various studies have documented that VATS can confer the similar capability of mediastinal lymph node dissection compared to thoracotomy, including number of dissections and the number of groups (21). Here, we found that RATS and axillary thoracotomy generated same outcome of lymph node dissection, suggested that surgical robot can thoroughly dissect the lymph nodes as thoracotomy does. So, further thoracotomy was unnecessary for dissection of mediastinal lymph nodes.

Unplanned intraoperative conversion to thoracotomy is an essential issue for the feasibility of RATS. The metaanalysis by Liang et al. (22) indicated that conversion rate to open surgery was significantly lower in patients who underwent RATS than those underwent VATS $[10.3 \%$ vs. $11.9 \%$; odds ratio (OR) $0.57, \mathrm{P}<0.001]$. In this trial, five subjects who initially underwent RATS were converted to axillary thoracotomy due to either of extensive pleural adhesion or equipment issues, but not due to intraoperative bleeding. The conversion to open surgery in this trial occupied $8.62 \%$ of cases, which was slightly lower earlier analysis. These findings suggested that unplanned intraoperative conversion would be rare event and should not withhold the surgeons from adopting the new techniques with complete establishment of learning curve for RATS lobectomy and standardized procedure.

To our knowledge, this phase 3 multicenter clinical trial is the first randomized study to evaluate the safety and short outcomes of RATS in treatment of cN2 NSCLC by comparing to axillary thoracotomy. It should be noticed that superiority of RATS for quicker recovery might be concealed in this study because the "length of stay" in this study did not reflect patients' true recovery from operation. Preoperative workups and assessment of protocol before operation took additional days and included in days of hospitalization. Also, analysis together data from five conversion subjects with other RATS patients did not change perioperative outcomes but should be negative factors for other outcomes, such as postoperative pain, acute inflammatory reaction, respiratory function, and quality of life. The "modified intension-to-treat analysis" that allow exclusion of the conversion patients for sub analysis of our clinical trial should be done in further.

In conclusion, RATS is safe and effective to treat patients with cN2 NSCLC owing to its similar shortterm outcomes of thorough dissection of lymph node and occurrence of postoperative complications compared to axillary thoracotomy and may be superior due to its lesser intraoperative blood loss. However, long-term follow-up is warranted to verify the superior or equivalent oncologic outcome of RATS lobectomy. The report could be expected by the end of 2020 .

\section{Acknowledgments}

Funding: This work was supported by Shanghai Hospital Development Center (Grant Number: SHDC12016113), National Natural Science Foundation of China (No. 81702251).

\section{Footnote}

Conflicts of Interest: The authors have no conflicts of interest to declare.

Ethical Statement: The authors are accountable for all aspects of the work in ensuring that questions related to the accuracy or integrity of any part of the work are appropriately investigated and resolved. The ethics board approved the study of Shanghai Chest Hospital (KS1735). Written informed consent was obtained from the patient for publication of this manuscript and any accompanying images.

\section{References}

1. Melfi FM, Menconi GF, Mariani AM, et al. Early experience with robotic technology for thoracoscopic surgery. Eur J Cardiothorac Surg 2002;21:864-8.

2. Melfi FM, Ambrogi MC, Lucchi M, et al. Video robotic lobectomy. Multimedia Man Cardiothorac Surg 2005;2005:mmcts.2004.000448. 
3. Kim HK, Choi YS, Kim J, et al. Outcomes of unexpected pathologic N1 and N2 disease after video-assisted thoracic surgery lobectomy for clinical stage I non-small cell lung cancer. J Thorac Cardiovasc Surg 2010;140:1288-93.

4. Greene FL, Page DL, Fleming ID, et al. Lung. In: AJCC Cancer Staging Manual. 6th ed. New York, NY: Springer; 2002:165-77.

5. Branch SK. Guidelines from the International Conference on Harmonisation (ICH). J Pharm Biomed Anal 2005;38:798-805.

6. Swanson SJ, Herndon JE 2nd, D'Amico TA, et al. Videoassisted thoracic surgery lobectomy: report of CALGB 39802-a prospective, multi-institution feasibility study. J Clin Oncol 2007;25:4993-7.

7. Giulianotti PC, Coratti A, Angelini M, et al. Robotics in general surgery: personal experience in a large community hospital. Arch Surg 2003;138:777-84.

8. Park BJ, Flores RM, Rusch VW. Robotic assistance for video-assisted thoracic surgical lobectomy: technique and initial results. J Thorac Cardiovasc Surg 2006;131:54-9.

9. Gharagozloo F, Margolis M, Tempesta B, et al. Robotassisted lobectomy for early-stage lung cancer: report of 100 consecutive cases. Ann Thorac Surg 2009;88:380-4.

10. Veronesi G, Galetta D, Maisonneuve P, et al. Four-arm robotic lobectomy for the treatment of early-stage lung cancer. J Thorac Cardiovasc Surg 2010;140:19-25.

11. Augustin F, Bodner J, Wykypiel H, et al. Initial experience with robotic lung lobectomy: report of two different approaches. Surg Endosc 2011;25:108-13.

12. Bodner J, Wykypiel H, Wetscher G, et al. First experiences with the da Vinci operating robot in thoracic surgery. Eur J Cardiothorac Surg 2004;25:844-51.

13. Zhao X, Qian L, Lin H, et al. Robot-assisted lobectomy for nonsmall cell lung cancer in China: initial experience and techniques. J Thorac Dis 2010;2:26-8.

14. Melfi FM, Mussi A. Robotically assisted lobectomy: learning curve andcomplications. Thorac Surg Clin 2008;18:289-95;vi-vii.

15. Anderson CA, Hellan M, Falebella A, et al. Roboticassisted lung resection for malignant disease. Innovations (Phila) 2007;2:254-8.

16. Giulianotti PC, Buchs NC, Caravaglios G, et al. Robotassisted lung resection: outcomes and technical details. Interact Cardiovasc Thorac Surg 2010;11:388-92.

17. Park BJ, Yang HX, Woo KM, et al. Minimally invasive (robotic assisted thoracic surgery and video-assisted thoracic surgery) lobectomy for the treatment of locally advanced non-small cell lung cancer. J Thorac Dis 2016;8:S406-13.

18. Cerfolio RJ, Bryant AS, Skylizard L, et al. Initial consecutive experience of completely portal robotic pulmonary resection with 4 arms. J Thorac Cardiovasc Surg 2011;142:740-6.

19. Hoksch B, Ablassmaler B, Walter M, et al. Radical thoracoscopic lobectomy with lymphadenectomy in a cadaver model. Can J Surg 2002;45:376-80.

20. Sagawa M, Sato M, Sakurada A, et al. A prospective trial of systematic nodal dissection for lung cancer by videoassisted thoracic surgery: can it be perfect. Ann Thorac Surg 2002;73:900-4.

21. Watanabe A, Mishina T, Ohori S, et al. Is video-assisted thoracoscopic surgery a feasible approach for clinical $\mathrm{NO}$ and postoperatively pathological N2 non-small cell lung cancer. Eur J Cardiothorac Surg 2008;33:812-8.

22. Liang H, Liang W, Zhao L, et al. Robotic Versus Videoassisted Lobectomy/Segmentectomy for Lung Cancer: A Meta-analysis. Ann Surg 2018;268:254-9.

Cite this article as: Huang J, Li C, Li H, Lv F, Jiang L, Lin H, Lu P, Luo Q, Xu W. Robot-assisted thoracoscopic surgery versus thoracotomy for c-N2 stage NSCLC: short-term outcomes of a randomized trial. Transl Lung Cancer Res 2019;8(6):951-958. doi:10.21037/tlcr.2019.11.31 